\title{
Expression Patterns and Correlations with Metabolic Markers of Zinc Transporters ZIP14 and ZNT1 in Obesity and Polycystic Ovary Syndrome
}

\begin{abstract}
Trine Maxel' ${ }^{\text {* }}$ Pernille Fog Svendsen ${ }^{2}$ Kamille Smidt ${ }^{3}$, Jesper Krogh Lauridsen", Birgitte Brock ${ }^{4}$, Steen Bønlykke Pedersen ${ }^{3,5}$, Jørgen Rungby ${ }^{6}$ and Agnete Larsen ${ }^{1}$

${ }^{1}$ Faculty of Health, Department of Biomedicine, Aarhus University, Aarhus, Denmark, ${ }^{2}$ Department of Obstetrics and Gynecology, Herlev University Hospital, Herlev, Denmark, ${ }^{3}$ Faculty of Health, Department of Clinical Medicine, Aarhus University, Aarhus, Denmark, ${ }^{4}$ Department of Clinical Biochemistry, Aarhus University Hospital, Aarhus, Denmark, ${ }^{5}$ Department of Endocrinology (MEA), Aarhus University Hospital, Aarhus, Denmark, ${ }^{6}$ Center for Diabetes Research, Department of Medicine, Gentofte University Hospital, Hellerup, Denmark
\end{abstract}

OPEN ACCESS

Edited by:

Ralf Jockers,

University of Paris, France

Reviewed by:

Rostislav Turecek,

Academy of Sciences of Czech

Republic, Czech Republic

Livio Casarini,

University of Modena and Reggio Emilia, Italy

*Correspondence: Trine Maxel tmj@biomed.au.dk

Specialty section: This article was submitted to Cellular Endocrinology,

a section of the journal

Frontiers in Endocrinology

Received: 04 July 2016 Accepted: 14 February 2017 Published: 02 March 2017

Citation: Maxel T, Svendsen PF, Smidt K, Lauridsen JK, Brock B, Pedersen SB,

Rungby J and Larsen A (2017)

Expression Patterns and Correlations with Metabolic Markers of Zinc

Transporters ZIP14 and ZNT1 in

Obesity and Polycystic

Ovary Syndrome.

Front. Endocrinol. 8:38.

doi: 10.3389/fendo.2017.00038
Polycystic ovary syndrome (PCOS) is associated with infertility, increased androgen levels, and insulin resistance. In adipose tissue, zinc facilitates insulin signaling. Circulating zinc levels are altered in obesity, diabetes, and PCOS; and zinc supplementation can ameliorate metabolic disturbances in PCOS. In adipose tissue, expression of zinc influx transporter ZIP14 varies with body mass index (BMI), clinical markers of metabolic syndrome, and peroxisome proliferator-activated receptor gamma (PPARG). In this study, we investigated expression levels of ZIP14 and PPARG in subcutaneous adipose tissue of 36 PCOS women (17 lean and 19 obese women) compared with 23 healthy controls (7 lean and 16 obese women). Further, expression levels of zinc transporter ZIP9, a recently identified androgen receptor, and zinc efflux transporter ZNT1 were investigated, alongside lipid profile and markers of glucose metabolism [insulin degrading enzyme, retinol-binding protein 4 (RBP4), and glucose transporter 4 (GLUT4)]. We find that ZIP14 expression is reduced in obesity and positively correlates with PPARG expression, which is downregulated with increasing BMI. ZNT1 is upregulated in obesity, and both ZIP14 and ZNT1 expression significantly correlates with clinical markers of altered glucose metabolism. In addition, RBP4 and GLUT4 associate with obesity, but an association with PCOS as such was present only for PPARG and RBP4. ZIP14 and ZNT1 does not relate to clinical androgen status and ZIP9 is unaffected by all parameters investigated. In conclusion, our findings support the existence of a zinc dyshomeostasis in adipose tissue in metabolic disturbances including PCOS-related obesity.

Keywords: obesity, polycystic ovary syndrome, peroxisome proliferator-activated receptor gamma, SIc30a, SIc39a, ZIP14, ZNT1, zinc

Abbreviations: BMI, body mass index; DHT, dihydrotestosterone; GLUT4, glucose transporter 4; HDL, high-density lipoprotein; HOMA index, homeostatic model assessment; IDE, insulin degrading enzyme; LDL, low-density lipoprotein; LRP10, lowdensity lipoprotein receptor-related protein-10; PCOS, polycystic ovary syndrome; PPARG, peroxisome proliferator-activated receptor gamma; RBP4, retinol-binding protein 4; SHBG, sex hormone-binding globulin; VLDL, very-low-density lipoprotein. 


\section{INTRODUCTION}

Polycystic ovary syndrome (PCOS) is the most common cause of infertility, affecting approximately $12-18 \%$ of all women of reproductive age (1). The syndrome is linked to increased risk of cardiovascular disease and type 2 diabetes (2). High androgen levels and oligo/anovulation are characteristics of PCOS, as are metabolic disturbances such as insulin resistance, visceral obesity, and dyslipidemia (2).

Altered serum-zinc levels are found in women with PCOS compared to body weight-matched, healthy controls, and both increased and decreased zinc levels have been reported (3-5). Interestingly, 8 weeks of zinc supplementation has beneficial effects on fasting plasma glucose, insulin resistance as measured by homeostatic model assessment (HOMA index), and the lipid profile in PCOS women (6). Similar to PCOS, a disturbed zinc homeostasis is also common in type 2 diabetic and obese individuals, which is evident by the presence of hypozincemia $(7-10)$. Low zinc intake in obese individuals has been linked to hyperinsulinemia, increased low-grade inflammation, and an aggravated lipid profile (11). These results indicate the presence of a disturbed zinc homeostasis in PCOS women, potentially linked with their obesity, and with an adverse effect on their metabolic health.

The essential trace metal zinc is required for the function of more than 300 enzymes and with an effect on at least 2,000 transcription factors (12). Free cytoplasmic zinc also regulates gene expression through the metal-responsive transcription factor-1 (13) and the level of free zinc can affect intracellular signaling pathways, e.g., by inhibition of protein tyrosine phosphatase (14). Due to its intracellular signaling and structural functions, zinc plays a role in lipid and glucose metabolism as well as in fertility $(12,15)$. Zinc can activate the insulin signaling pathway through inhibition of protein tyrosine phosphatase leading to increased phosphorylation of the insulin receptor (14). Further, zinc ions can work in an insulinomimetic way within adipocytes, stimulating lipogenesis and glucose transport through translocation of glucose transporter 4 (GLUT4) to the cell membrane $(16,17)$. In the reproductive system, zinc deficiency associates with poor pregnancy outcomes and zinc is crucial for normal sexual development in both men and women $(12,18-21)$. Animal studies show that zinc deficiency affects ovulation, as well as zinc has an effect on the estrogen level and menstrual cycle $(22,23)$. In men, zinc deficiency is linked to decreased circulating levels of testosterone and dihydrotestosterone (DHT) (23).

The regulation of intracellular zinc homeostasis is maintained by a number of zinc transporters and metallothioneins $(24,25)$. Fourteen human zinc influx transporters (SLC39a; ZIP1-14) and 10 zinc-efflux transporters (SLC30a; ZNT1-10) have so far been identified $(24,25)$.

The zinc influx transporter ZIP14 is a member of the LIV-1 subfamily of zinc transporters that localize to the plasma membrane and facilitate the transport of zinc into the cell (26). ZIP14 is believed to be important in adipogenesis (27-29). We recently reported that ZIP14 expression in adipose tissue is reversibly downregulated by obesity and inversely correlated with clinical markers of metabolic disease (27). Further, we found a possible link between ZIP14 expression and the transcription factor peroxisome proliferator-activated receptor gamma (PPARG) in humans (27), a finding also seen in studies of adipose tissue from Zip14 knockout mice (29). PPARG regulates lipid uptake and glucose metabolism, and plays an essential role in adipogenesis (30). Polymorphisms in the PPARG gene have been shown to decrease the risk and affect the metabolic phenotype of PCOS $(31,32)$ and the function of PPARG is regulated by zinc status $(33,34)$. Similar to other members of the LIV-1 subfamily of zinc transporters, estrogen can induce zinc influx through ZIP14 $(26,35,36)$ and another zinc influx transporter, ZIP9, has recently been identified as an androgen receptor in human hormone-responsive breast and prostate cancer cells, with androgens stimulating ZIP9mediated zinc influx $(37,38)$.

In this study, we aimed at investigating if intracellular zinc dyshomeostasis, as reflected by changes in expression of zinc transporters, is directly linked to the PCOS syndrome. Specifically, we aimed at investigating the expression levels of ZIP14, due to its indicated role in adipose tissue functioning and association with metabolic disease. A potential sex-hormonal and metabolic regulation of ZIP14, as a member of the LIV-1 subfamily, were investigated together with the expression levels of the androgen receptor ZIP9 and the main zinc efflux transporter ZNT1, in adipose tissue from lean and obese women with PCOS as well as weight-matched controls. The expression levels were correlated with clinical markers of lipid and glucose metabolism, androgen status, as well as expression of genes related to glucose metabolism within adipose tissue, i.e., GLUT4, insulin degrading enzyme (IDE), and retinol-binding protein $4(R B P 4)$, all speculated to be involved in metabolic disease.

\section{MATERIALS AND METHODS}

\section{Participants and Interventions}

Thirty-six lean [body mass index (BMI) $=18.5-25 ; n=17$ ] and obese (BMI $>25 ; n=19)$ women with PCOS, and 23 agematched lean $(n=7)$ and obese $(n=16)$ women without PCOS (control), recruited through advertising in the local newspaper, were included in the study. The women in the control group had a regular menstrual cycle ( $<35$ days) and an androgen level within the normal range. None of the participants suffered from other chronic diseases or used oral contraceptives or any other drugs known to alter glucose or insulin metabolism within the last 3 months prior to the study. PCOS was diagnosed according to the Rotterdam criteria (39), i.e., based on the androgen level, evaluation of hirsutism (Ferriman-Gallwey score $\geq 8$ ), transvaginal ultrasonography, and menstrual history (40). The study design and assessment of clinical parameters (PCOS status, anthropometrics, insulin and glucose measurements, lipid profile, and hormone analysis) have been previously described (40-44). The study was performed at the Department of Endocrinology and Department of Obstetrics and Gynecology, Hvidovre University 
Hospital, Hvidovre, Denmark. The study was approved by the local ethics authorities and followed the principles in the "Declaration of Helsinki." All the subjects gave their written informed consent prior to the study.

\section{Determination of Body Fat Percentage and Measurement of Glucose Metabolism, Lipid Profile, and Sex Hormones}

Participants underwent a dual-emission X-ray absorptiometry scan to determine the body fat percentage. Subcutaneous fat biopsies for gene expression studies were obtained from the lower abdomen. Blood samples were collected following an overnight fasting. Plasma glucose was measured using a Beckman Glucose analyzer (Ramcon, Fullerton, CA, USA), and serum insulin was determined using the 1235 Auto DELPHIA Automatic Immunoassay System (Wallac Oy, Turku, Finland). The HOMA index was calculated as follows: fasting serum insulin concentration $(\mu \mathrm{U} / \mathrm{L}) \times$ fasting plasma glucose concentration $(\mathrm{mmol} / \mathrm{L}) / 22.5(45,46)$. Fasting levels of cholesterol, high-density lipoprotein (HDL), low-density lipoprotein, very-low-density lipoprotein (VLDL), and triglycerides were measured by reflection photometry (Ortho-Clinical diagnostics kit, Raritan, NJ, USA). Testosterone, DHT, sex hormone-binding globulin (SHBG), and the calculation of free testosterone were performed as previously described using immunofluorometric assays and radioimmunoassays (40).

\section{Real-time Polymerase Chain Reaction (PCR)}

RNA was extracted from the fat biopsies using TRIzol (Gibco BRL, Life Technologies, Roskilde, Denmark). The concentration and quality of the purified total RNA were determined spectrophotometrically and by running an agarose gel. Reverse transcription was performed using the Thermo Scientific Verso cDNA kit (Applied Biosystems, Foster City, CA, USA). The KAPA SYBR FAST qPCR kit (Kapa Biosystems, Inc., Woburn, MA, USA) and a LightCycler 480 (Roche Applied Science, Basel, Switzerland) were used for the real-time PCRs. Duplicate reactions of all samples were performed. The expression levels of ZIP14, ZIP9, ZNT1, PPARG1, PPARG2, IDE, RBP4, and GLUT4 were measured. Gene accession numbers and primers are listed in Table S1 in Supplementary Material. Low-density lipoprotein receptor-related protein-10 (LRP10) was used as a housekeeping gene, as it shows a stable expression in human adipose tissue (47). The stability of $L R P 10$ was confirmed by statistically comparing $C_{\mathrm{t}}$-cycles between groups. Relative expression levels were calculated using the Advanced Relative Quantification mode of the LightCycler 480 instrument, version 1.5.0.39 (Roche Applied Science).

\section{Statistical Analysis}

Metabolic data and androgen status are presented as the means $\pm S D$, and the groups were compared by Student's $t$-test. Data were found to be normally distributed (QQ plots), except VLDL and HOMA index values which were logtransformed to obtain a normal distribution. Real-time PCR data are presented as the mean starting quantity of (gene of interest/LRP10) \pm SEM. Statistical analyses of the PCR results were performed on log-transformed data to obtain a normal distribution. The influence of PCOS and obesity and their interaction with regards to the expression of the genes were examined by two-way ANOVA analysis. In the presence of a statistically detectable interaction, a combined (interfering) effect exists in which the relationship between a factor, e.g., weight status and the effect on gene expression differs by the presence of the other factor, e.g., PCOS. If statistically significant differences were found among the four groups, subgroup analyses were performed using Student's $t$-test in order to identify the origins of this difference; e.g., following a significant two-way ANOVA showing an effect of obesity, obese PCOS women vs. lean PCOS women, and obese control women vs. lean control women were compared. Correlations included the whole study population $(n=59)$ and were performed using a standard Pearson's correlation and log-transformed PCR data. Although specifically for PPARG1, correlations were additionally done separately for PCOS and non-PCOS women, as PPARG1 showed a regulation by weight and PCOS status. The level of statistical significance in all analyses was set at 0.05. STATA (StataCorp LP, TX, USA) and GraphPad Prism 5 (GraphPad Software Inc., La Jolla, CA, USA) statistical packages were used for the calculations.

\section{RESULTS}

\section{Metabolic Measurements and Androgen Status of PCOS Women and Controls}

Metabolic and androgen measurements were performed on all subjects to establish their metabolic status and to confirm the presence or absence of PCOS pathology.

Anthropometric findings, insulin and glucose measurements, the lipid profile, and the results of the hormone analysis are shown in Table 1. For further details on the metabolic and sex-hormonal measurements, please refer to Ref. $(40,41,43,44)$. The age was comparable between groups (40). In addition, BMI was similar between lean PCOS women and lean controls, and between obese PCOS women and obese controls $(p=0.1707$ and $p=0.2482$, respectively). As expected, the HOMA index was significantly higher in the obese women than that in lean women in both the PCOS and control groups $(p=0.0002$ and $p<0.0001$, respectively). The total testosterone level was significantly increased in the PCOS women compared to the control women in both the lean and obese groups ( $p=0.01$ and $p<0.0001$, respectively) $(40,41,43,44)$.

In summary, we confirmed the expected differences in BMI, HOMA index, and androgen levels of the four groups (lean and obese women with and without PCOS), establishing that age did not differ significantly among groups nor did BMI differ between the obese controls and the obese PCOS women or between lean controls and lean PCOS women. 
TABLE 1 | Metabolic measurements and androgen status of lean and obese women with PCOS, and lean and obese control women without PCOS.

\begin{tabular}{|c|c|c|c|c|}
\hline & \multicolumn{2}{|c|}{ PCOS } & \multicolumn{2}{|c|}{ Controls } \\
\hline & $\begin{array}{l}\text { Lean PCOS } \\
\quad(n=17)\end{array}$ & $\begin{array}{l}\text { Obese } \\
\text { PCos } \\
(n=19)\end{array}$ & $\begin{array}{l}\text { Lean controls } \\
\qquad(n=7)\end{array}$ & $\begin{array}{c}\text { Obese } \\
\text { controls } \\
(n=16)\end{array}$ \\
\hline Age (years) & $28( \pm 5)$ & $29( \pm 4)$ & $30( \pm 4)$ & $31( \pm 5)$ \\
\hline BMI (kg/m²) & $23( \pm 2) \bullet \bullet \bullet$ & $33( \pm 4)$ & $22( \pm 2) \bullet \bullet \bullet$ & $34( \pm 3)$ \\
\hline Body fat (\%) & $27( \pm 4)^{\bullet \bullet \bullet}$ & $41( \pm 7)$ & $24( \pm 7) \bullet \bullet$ & $43( \pm 6)$ \\
\hline Insulin (pmol/l) & $36( \pm 18) \bullet \bullet$ & $67( \pm 39)$ & $25( \pm 14)^{\bullet \bullet}$ & $64( \pm 31)$ \\
\hline Glucose $(\mathrm{mmol} / \mathrm{l})$ & $5.5( \pm 0.4)^{\bullet}$ & $6.0( \pm 0.6)^{\circ}$ & $5.6( \pm 0.4)$ & $5.6( \pm 0.3)$ \\
\hline HOMA-IR & $1.5( \pm 0.8) \bullet \bullet \bullet$ & $3.0( \pm 1.8)$ & $1.0( \pm 0.6) \bullet \bullet \bullet$ & $2.7( \pm 1.3)$ \\
\hline HDL (mmol/l) & $1.4( \pm 0.3)^{0} \bullet \bullet \bullet$ & $1.1( \pm 0.2)$ & $1.1( \pm 0.2)$ & $1.1( \pm 0.2)$ \\
\hline VLDL (mmol/l) & $0.4( \pm 0.2)$ & $0.4( \pm 0.2)$ & $0.4( \pm 0.1)$ & $0.6( \pm 0.3)$ \\
\hline $\begin{array}{l}\text { Cholesterol } \\
(\mathrm{mmol} / \mathrm{l})\end{array}$ & $4.3( \pm 1.0)$ & $4.2( \pm 0.6)$ & $3.6( \pm 0.6)^{\bullet \bullet}$ & $4.7( \pm 0.8)$ \\
\hline $\begin{array}{l}\text { Triglycerides } \\
(\mathrm{mmol} / \mathrm{l})\end{array}$ & $0.8( \pm 0.4)$ & $1.0( \pm 0.3)$ & $0.8( \pm 0.2)^{\bullet}$ & $1.2( \pm 0.6)$ \\
\hline $\begin{array}{l}\text { T-testosterone } \\
(\mathrm{nmol} / \mathrm{l})\end{array}$ & $2.1( \pm 0.8)^{\circ}$ & $2.4( \pm 0.8)^{\circ 00}$ & $1.4( \pm 0.3)$ & $1.4( \pm 0.4)$ \\
\hline $\begin{array}{l}\text { F-testosterone } \\
(\mathrm{nmol} / \mathrm{l})\end{array}$ & $\begin{array}{c}0.034 \\
( \pm 0.019)^{\circ 0}\end{array}$ & $\begin{array}{c}0.043 \\
( \pm 0.022)^{\circ \circ}\end{array}$ & $\begin{array}{c}0.015 \\
( \pm 0.006)^{\bullet}\end{array}$ & $\begin{array}{c}0.024 \\
( \pm 0.007)\end{array}$ \\
\hline $\mathrm{SHBG}(\mathrm{nmol} / \mathrm{l})$ & $67( \pm 27)^{\circ}$ & $59( \pm 39)$ & $101( \pm 38) \bullet \bullet$ & $54( \pm 21)$ \\
\hline
\end{tabular}

Data are presented as the means $\pm S D$.

$B M I$, body mass index; F-testosterone, free testosterone; HDL, high-density lipoprotein; HOMA-IR, homeostatic model assessment-insulin resistance; PCOS,

polycystic ovary syndrome; SHBG, sex hormone-binding globulin; T-testosterone, total testosterone; VLDL, very-low-density lipoprotein.

$\circ p<0.05,0^{\circ} p<0.01,000 p<0.001$ comparing lean PCOS subjects vs. lean controls or obese PCOS subjects vs. obese controls using Student's t-test.

$\bullet p<0.05, \bullet p<0.01, \bullet \bullet<0.001$ comparing lean PCOS subjects vs. obese PCOS subjects or lean controls vs. obese controls.

Level of statistical significance: 0.05

\section{ZIP14 and ZNT1 Expression Are Oppositely Regulated in Obesity but Appear Unaffected by PCOS Status, While ZIP9 Expression in Adipose Tissue Is Unaffected in Both Obesity and PCOS}

Adipose expression levels of ZIP14, ZIP9, and ZNT1 were investigated in order to identify if their gene expression is regulated by obesity and/or PCOS status, as we have previously reported a regulation of obesity on adipose ZIP14 expression (27).

Zinc influx transporter ZIP14 and zinc efflux transporter ZNT1 expression were oppositely regulated in obese women. ZIP14 expression decreased significantly in obese individuals compared with lean individuals by two-way ANOVA analysis ( $p<0.0001$; subgroup analysis examining lean PCOS vs. obese PCOS, and lean controls vs. obese controls using Student's $t$-test; $p=0.0008$ and $p=0.0079$, respectively). Neither PCOS status $(p=0.3892)$ nor any combinatory influence of PCOS and weight status had a significant effect on ZIP14 expression by two-way ANOVA analysis ( $p=0.6908)$ (Figure 1A).

Obesity showed a significant upregulatory effect on ZNT1 expression by two-way ANOVA analysis $(p=0.0177$; subgroup analysis examining lean PCOS vs. obese PCOS, and lean controls vs. obese controls using Student's $t$-test; $p=0.0169$ and $p=0.2489$, respectively). However, PCOS status did not relate to ZNT1 expression, and no combinatory effect between PCOS status and weight was found with regards to ZNT1 expression levels using two-way ANOVA analysis $(p=0.3577$ and $p=0.6396$, respectively) (Figure 1B).

ZIP9 expression was unaffected by both obesity and PCOS status, and no combinatory effect between the two parameters was seen by two-way ANOVA analysis $(p=0.4764, p=0.8036$, and $p=0.3516$, respectively) (Figure 1C).

In conclusion, ZIP14 and ZNT1 were oppositely regulated by obesity, with a downregulation of ZIP14 and upregulation of $Z N T 1$, but the adipose expression of these zinc transporters was not significantly altered by PCOS status.

\section{PPARG1 Expression in Adipose Tissue Is Downregulated by Both Obesity and PCOS}

With a known function in lipid and glucose metabolism as well as a role in adipogenesis, adipose PPARG expression levels (both isoforms 1 and 2) were investigated in terms of a potential regulation of these genes by PCOS status alone or in combination with obesity.

The mRNA isoforms PPARG1 and PPARG2 were investigated. Two-way ANOVA analysis showed a significant downregulation of PPARG1 expression by obesity $(p<0.0001$; subgroup analysis examining lean PCOS vs. obese PCOS, and lean controls vs. obese controls using Student's $t$-test; $p=0.0001$ and $p<0.0001$, respectively). In addition, twoway ANOVA testing showed a significant downregulatory effect of PCOS on PPARG1 expression ( $p=0.0291$; subgroup analysis examining lean PCOS vs. lean controls, and obese PCOS vs. obese controls using Student's $t$-test; $p=0.0045$ and $p=0.5380$, respectively). However, no additional combined effect of PCOS and weight status was found with regards to PPARG1 expression ( $p=0.1739)$ (Figure 2A). Two-way ANOVA showed no significant effect of weight or PCOS status, nor any combined effect of the two on PPARG2 expression ( $p=0.2021, p=0.1244$, and $p=0.0701$, respectively). Notably, mean expression levels were highest in lean controls, as found for PPARG1 (Figure 2B).

In summary, the expression of PPARG1 was significantly downregulated by the presence of PCOS as well as by the presence of obesity. However, the combined effect of PCOS and obesity did not statistically differ from the presence of just one of these conditions. Statistically, PPARG2 showed no regulation by PCOS or obesity status, but showed a similar expression pattern to that of PPARG1 with a high expression in lean controls compared to the other groups, indicating a potential negative effect of PCOS and obesity.

\section{Expression of ZIP14 in Adipose Tissue Is Correlated with PPARG, BMI, and Body Fat Percentage}

As ZIP14 expression levels were found to be regulated by obesity and ZIP14 is believed to play a role in adipogenesis, the association with the measured metabolic markers was investigated in terms 

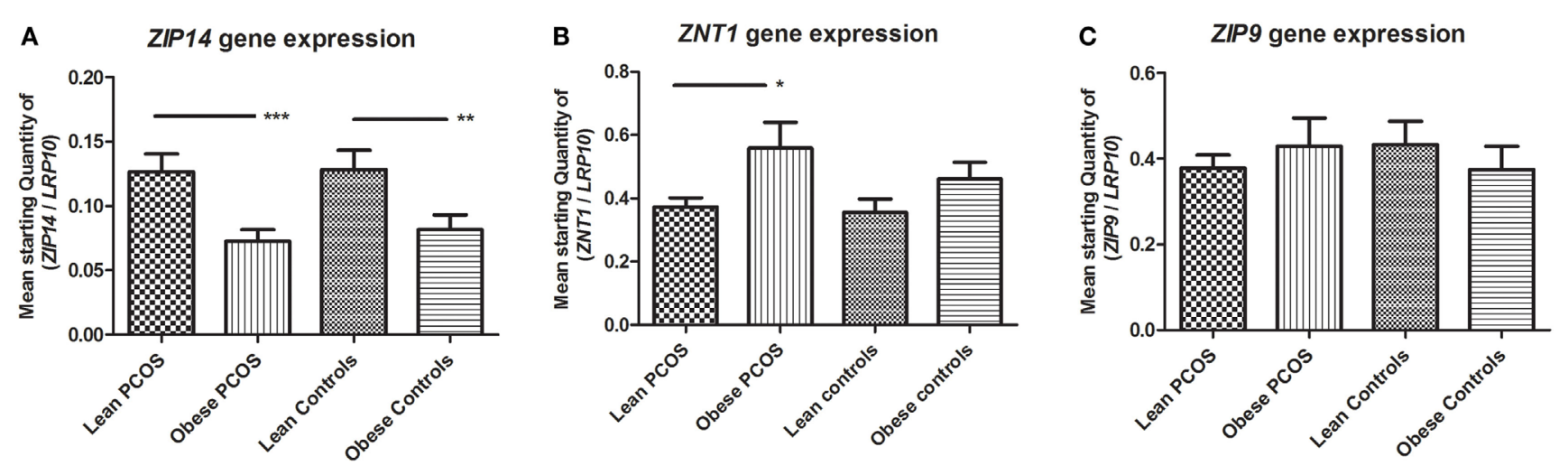

FIGURE 1 | Expression of zinc transporters ZIP14, ZNT1, and ZIP9 in adipose tissue from lean and obese women with polycystic ovary syndrome (PCOS), and from lean and obese control women without PCOS. Results are expressed as the mean starting quantity of (gene of interest/LRP10) \pm SEM. (A) Expression of ZIP14. (B) Expression of ZNT1. (C) Expression of ZIP9. ${ }^{\star} p<0.05$; ${ }^{\star \star} p<0.01$; ${ }^{\star \star \star} p<0.001$. LRP10, low-density lipoprotein receptor-related protein-10. Lean PCOS women, $n=17$; obese PCOS women, $n=19$; lean control women, $n=7$; obese control women, $n=16$. The groups were compared by two-way ANOVA analysis followed by subgroup analysis by Student's $t$-test if significant. Level of statistical significance: 0.05.
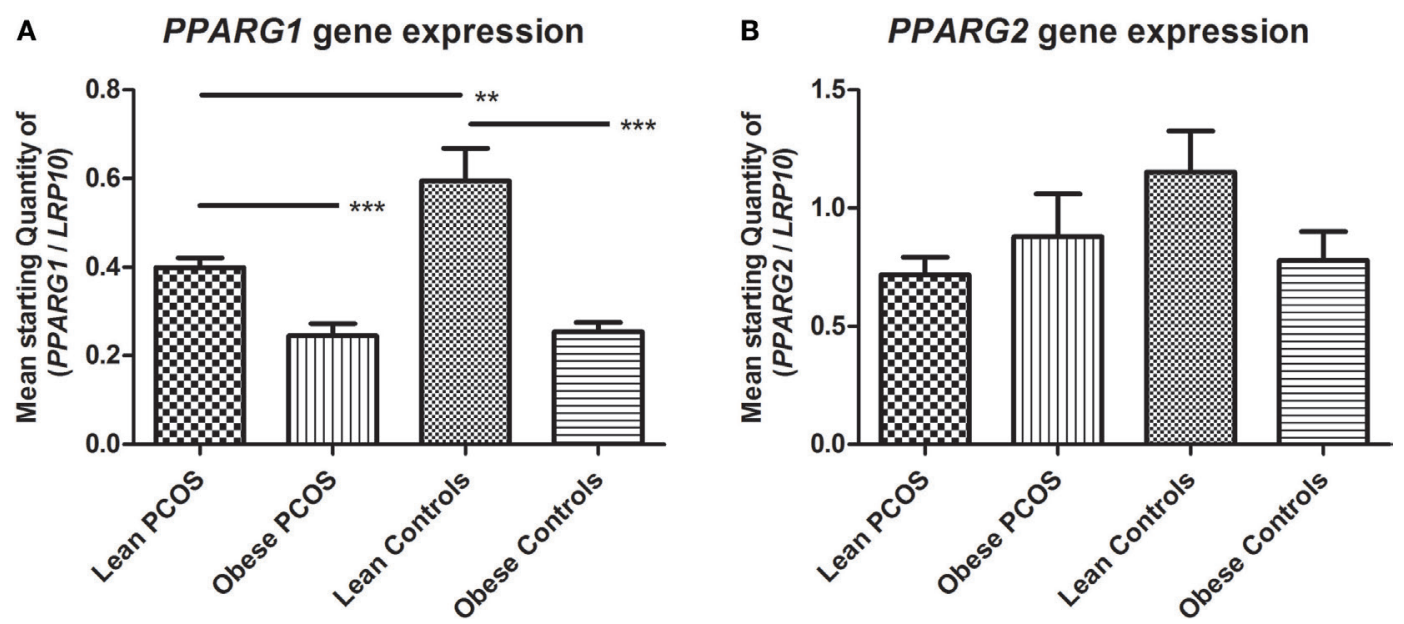

FIGURE 2 | Expression of peroxisome proliferator-activated receptor gamma (PPARG) in adipose tissue from lean and obese women with polycystic ovary syndrome (PCOS), and from lean and obese control women without PCOS. Results are expressed as the mean starting quantity of (gene of interest/LRP10) \pm SEM. (A) Expression of PPARG1. (B) Expression of PPARG2. ${ }^{\star \star} p<0.01$; ${ }^{\star \star \star} p<0.001$. LRP10, low-density lipoprotein receptor-related protein-10; PPARG1, peroxisome proliferator-activated receptor gamma mRNA isoform 1; PPARG2, peroxisome proliferator-activated receptor gamma mRNA isoform 2. Lean PCOS women, $n=17$; obese PCOS women, $n=19$; lean control women, $n=7$; obese control women, $n=16$. The groups were compared by two-way ANOVA analysis followed by subgroup analysis by Student's $t$-test if significant. Level of statistical significance: 0.05 .

of the correlation between ZIP14 expression and anthropometric measurements, lipid profile, and adipogenesis marker $P P A R G$ using Pearson's correlation.

ZIP14 expression associated closely with that of PPARG, with a significant positive correlation between the two genes, for both PPARG1 and PPARG2 levels $(p=0.0001$, and $p=0.0046$, respectively). When investigating the correlation between PPARG1 and ZIP14 expression separately for PCOS and non-PCOS women, as PPARG1 was shown to be statistically independently affected by PCOS status, similar correlations were found (PCOS: $r=0.49$, $p=0.0022$; non-PCOS: $r=0.48, p=0.0210$ ).
ZIP14 expression showed a strong inverse correlation with the anthropometric markers BMI $(p=0.0001)$ and body fat percentage $(p=0.0027)$. With regard to the lipid profile, ZIP14 expression showed a positive correlation with the HDL level ( $p=0.0421$ ), but no significant correlations were found with other lipid profile markers (VLDL, triglyceride, or cholesterol levels). All $r$-values and $p$-values are shown in Table 2.

In summary, ZIP14 showed a close inverse relationship with anthropometric markers of obesity as well as a positive association with the adipose expression of PPARG. 


\section{ZIP14 Expression in Adipose Tissue Is Significantly Correlated with Several Markers of Glucose Metabolism in Blood As Well As in Adipose Tissue, but Not with PCOS Markers}

As ZIP14 expression was found to be regulated by obesity, in which alterations in glucose homeostasis are found, we tested a potential association between ZIP14 expression and glucose homeostatic markers. Further we aimed at confirming that ZIP14 expression levels do not associate with PCOS status by investigating the association between ZIP14 expression and specific PCOS markers.

With regard to glucose metabolism, ZIP14 inversely correlated with fasting glucose levels $(p=0.0120)$. However, ZIP14 showed no significant correlation with fasting insulin levels or the HOMA index; although in the latter case, the correlation did approach significance $(p=0.0822)$. In adipose tissue, ZIP14 expression showed significant positive correlations with the glucose metabolic markers, RBP4 $(p=0.0117)$ and GLUT4 $(p=0.0009)$. ZIP14 expression showed no correlation with the PCOS indicators, namely, testosterone, SHBG, or the FerrimanGallwey score of hirsutism. All $r$-values and $p$-values are shown in Table 2.

In short, using a Pearson's correlation, ZIP14 showed an association with glucose homeostatic markers in adipose tissue and fasting glucose levels. No association was found with specific PCOS markers, confirming the statistical findings of the two-way ANOVA analysis.

TABLE 2 | Correlations among ZIP14 expression and anthropometric parameters, markers of lipid and glucose homeostasis, and markers of androgen function.

\begin{tabular}{ll}
\hline & ZIP14 expression \\
\hline PPARG1 & $r=0.48, p=0.0001$ \\
PPARG2 & $r=0.36, p=0.0046$ \\
BMI & $r=-0.47, p=0.0001$ \\
Body fat percentage & $r=-0.39, p=0.0027$ \\
Insulin & $r=-0.09, p=0.4955$ \\
Glucose & $r=-0.32, p=0.0120$ \\
HOMA-IR & $r=-0.23, p=0.0822$ \\
HDL & $r=0.27, p=0.0421$ \\
VLDL & $r=0.01, p=0.9238$ \\
Triglyceride & $r=-0.00, p=0.9748$ \\
Cholesterol & $r=-0.02, p=0.8888$ \\
T-testosterone & $r=-0.10, p=0.4617$ \\
F-testosterone & $r=-0.34, p=0.1070$ \\
SHBG & $r=0.16, p=0.2169$ \\
Ferriman-Gallwey & $r=-0.14, p=0.2849$ \\
RBP4 expression & $r=0.33, p=0.0117$ \\
GLUT4 expression & $r=0.42, p=0.0009$ \\
\hline
\end{tabular}

All women were included $(n=59)$ in this analysis. Pearson's $r$-values and $p$-values are indicated. Level of statistical significance: 0.05 .

BMI, body mass index; F-testosterone, free testosterone; GLUT4, glucose transporter 4; HDL, high-density lipoprotein; HOMA-IR, homeostatic model assessment-insulin resistance; PPARG, peroxisome proliferator-activated receptor gamma; RBP4, retinol-binding protein 4; SHBG, sex hormone-binding globulin; T-testosterone, total testosterone; and VLDL, very-low-density lipoprotein.

\section{ZNT1 Showed Associations That Were Opposite to Those of ZIP14 with BMI and Body Fat Percentage}

As the main zinc efflux transporter at the cell membrane, and due to its opposite regulation than ZIP14 by obesity, we investigated if ZNT1 expression was associated with the same parameters as ZIP14, in terms of anthropometric markers, lipid profile, and PPARG levels.

Using Pearson's correlation, ZNT1 showed associations that were opposite to those of ZIP14 with the measured anthropometric markers, as ZNT1 expression displayed a significant positive correlation with BMI $(p=0.0199)$ and body fat percentage $(p=0.0140)$. However, ZNT1 expression did not associate with the two PPARG isoforms (PPARG1, $p=0.2311 ;$ PPARG2, $p=0.1826)$, although when analyzing non-PCOS and PCOS women separately, as for ZIP14, a significant correlation with PPARG1 was observed in PCOS women $(r=-0.34$, $p=0.0401)$ but not in non-PCOS women $(r=0.13, p=0.5685)$. The correlation of ZNT1 expression with the lipid profile showed a negative association with $\operatorname{HDL}(p=0.0468)$. No other significant correlations were found. All $r$-values and $p$-values are shown in Table 3 .

In summary, ZNT1 showed an opposite association, to that of ZIP14, in terms of the anthropometric markers, but with no general association to PPARG expression levels, aside from the subgroup analysis of PCOS women only.

\section{Markers of Glucose Homeostasis Significantly Correlated with ZNT1 Expression}

As ZNT1 expression showed an opposite regulation by obesity than that of ZIP14, expression levels of ZNT1 were correlated

TABLE 3 | Correlations among ZNT1 expression and anthropometric parameters, markers of lipid and glucose homeostasis, and markers of androgen function.

\begin{tabular}{ll}
\hline & ZNT1 expression \\
\hline PPARG1 & $r=-0.16, p=0.2311$ \\
PPARG2 & $r=0.18, p=0.1826$ \\
BMI & $r=0.30, p=0.0199$ \\
Body fat percentage & $r=0.32, p=0.0140$ \\
Insulin & $r=0.29, p=0.0277$ \\
Glucose & $r=0.16, p=0.2268$ \\
HOMA-IR & $r=0.30, p=0.0213$ \\
HDL & $r=-0.26, p=0.0468$ \\
VLDL & $r=0.10, p=0.4634$ \\
Triglyceride & $r=0.15, p=0.2423$ \\
Cholesterol & $r=-0.01, p=0.9125$ \\
T-testosterone & $r=0.15, p=0.2423$ \\
F-testosterone & $r=0.03, p=0.8006$ \\
SHBG & $r=0.02, p=0.8886$ \\
Ferriman-Gallwey & $r=0.10, p=0.4377$ \\
RBP4 expression & $r=0.01, p=0.9437$ \\
GLUT4 expression & $r=0.05, p=0.6917$
\end{tabular}

All women $(n=59)$ were included in the analysis. Pearson's $r$-values and $p$-values are indicated. Level of statistical significance: 0.05 .

BMI, body mass index; F-testosterone, free testosterone; GLUT4, glucose transporter 4; HDL, high-density lipoprotein; HOMA-IR, homeostatic model assessment-insulin resistance; PPARG, peroxisome proliferator-activated receptor gamma; $R B P 4$, retinol-binding protein 4; SHBG, sex hormone-binding globulin; T-testosterone, total testosterone; and VLDL, very-low-density lipoprotein. 
with glucose homeostasis and markers specifically associated with PCOS, to investigate if ZNT1 associated with the same parameters as ZIP14 using a Pearson's correlation.

Of the markers of glucose homeostasis in the blood and adipose tissue, ZNT1 showed a significant positive correlation with the fasting insulin level $(p=0.0277)$ and the HOMA index $(p=0.0213)$. There was no statistical significant correlation of ZNT1 expression with fasting glucose levels or RBP4 and GLUT4 expression in adipose tissue. ZNT1 showed no statistical significant correlations with PCOS indicators, namely, testosterone, SHBG, or the Ferriman-Gallwey score of hirsutism. All $r$-values and $p$-values are shown in Table 3.

In summary, ZNT1 showed a correlation with blood glucose homeostatic markers, rather than markers of glucose homeostasis in adipose tissue. Confirming the results of the two-way ANOVA analysis, no association of ZNT1 expression was found with PCOS markers.

\section{RBP4 and GLUT4 Expression Is Downregulated in Adipose Tissue in Obese Women, with PCOS Status Affecting the RBP4 Level, While IDE Expression Is Unaffected by Obesity and PCOS Status}

As markers of glucose homeostasis within adipose tissue, RBP4, GLUT4, and IDE are all associated with the presence of insulin resistance. With insulin resistance being a feature of PCOS as well as a comorbid factor of obesity, we investigated if PCOS affect their expression in adipose tissue directly and if the presence of obesity can cause a similar change or affect any PCOS induced alterations in the expression of these genes.

A markedly higher RBP4 expression was seen in lean controls compared to the other three groups which appeared similar (lean and obese PCOS women as well as obese controls) (Figure 3A). Supporting this, two-way ANOVA confirmed a significant interaction between weight and PCOS status $(p=0.0185)$, reflecting that PCOS status plays a role on RPB4 expression and that this role depends on weight status, as no effect of PCOS per se was found ( $p=0.1544$, two-way ANOVA). In line with this, subgroup analysis using Student's $t$-test showed a significant difference between lean PCOS and lean controls ( $p=0.0170, t$-test), but not between obese PCOS and obese controls $(p=0.4188)$, as a downregulatory effect similar to the one of PCOS was found of obesity ( $p=0.0010$, two-way ANOVA). According to this, a significant difference was seen when comparing obese controls vs. lean controls ( $p=0.0002$, $t$-test) whereas no difference was found when comparing obese PCOS women vs. lean PCOS women $(p=0.4286$, $t$-test $)$.

As shown by the two-way ANOVA analysis, the adipose expression of GLUT4 was significantly downregulated by the presence of obesity ( $p=0.0009)$, evident in subgroup analysis using Student's $t$-test when comparing obese controls vs. lean controls ( $p=0.0185$, t-test) and tending toward statistically significance when comparing lean PCOS vs. obese PCOS ( $p=0.0547, t$-test). Two-way ANOVA analysis found no statistically significant effect of PCOS status on GLUT4 expression ( $p=0.1997$ ) nor any statistical interaction between PCOS status and obesity ( $p=0.1318$ ), although GLUT4 expression levels were higher in women in the lean control group than in any of the other three groups, as noted for $R B P 4$ (Figure 3B).

There were no statistical significant effects of PCOS status or obesity nor any interaction effect between the two on IDE expression levels by two-way ANOVA analysis $(p=0.9489, p=0.6998$ and $p=0.3055$, respectively) (Figure 3C).

In summary, for RBP4 expression, a high level was observed within the group of lean women compared to all other groups, which appeared similar, corresponding to a downregulatory response in lean PCOS women similar to the one observed
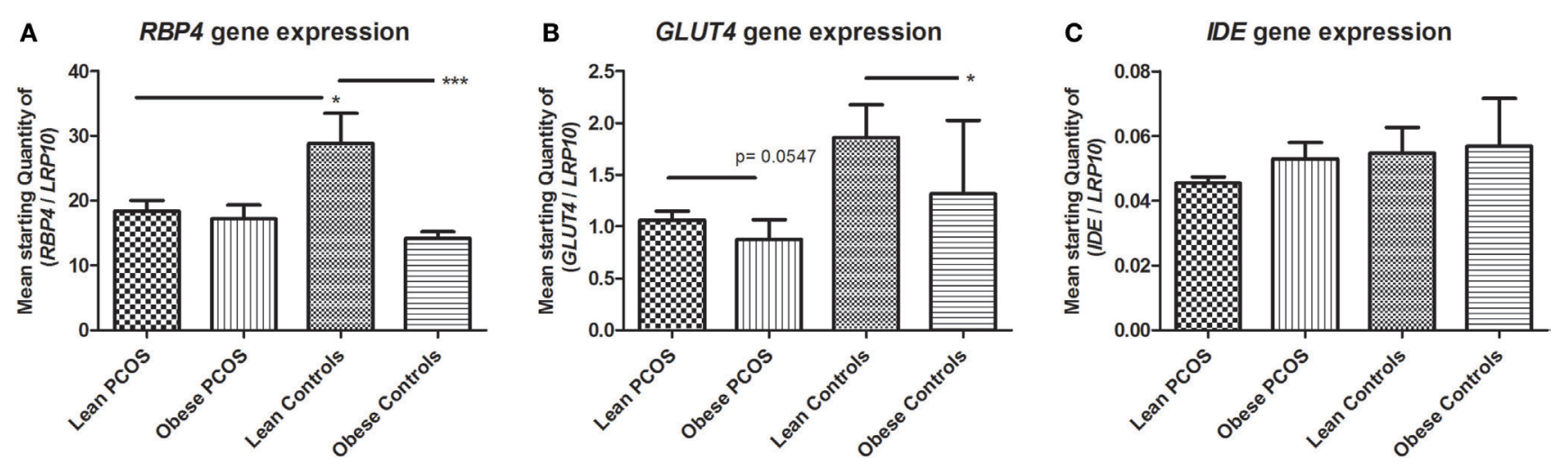

FIGURE 3 | Expression of markers of glucose metabolism in adipose tissue from lean and obese women with polycystic ovary syndrome (PCOS), and from lean and obese control women without PCOS. Results are expressed as the mean starting quantity of (gene of interest/LRP10) \pm SEM.

(A) Expression of RBP4. (B) Expression of GLUT4. (C) Expression of IDE. ${ }^{*} p<0.05$; ${ }^{* *} p<0.001$. GLUT4, glucose transporter 4; LRP10, low-density lipoprotein receptor-related protein-10; RBP4, retinol-binding protein 4; and IDE, insulin degrading enzyme. Lean PCOS women, $n=17$; obese PCOS women, $n=19$; lean control women, $n=7$; obese control women, $n=16$. The groups were compared by two-way ANOVA analysis followed by subgroup analysis by Student's $t$-test if significant. Level of statistical significance: 0.05 . 
when obese, and reflected as a statistically significant interaction between obesity and PCOS status. Likewise, expression of GLUT4 was high in lean women compared to the other groups, indicating a potential effect of obesity and PCOS status, although only statistically significant of obesity on GLUT4 expression.

\section{DISCUSSION}

Reduced levels of circulating zinc are common in obesity $(8,9)$ whereas recent studies report conflicting results with regard to the levels of circulating zinc in the blood of PCOS women (3-5). However, zinc supplementation has several beneficial effects on the metabolic status in PCOS women $(6,48)$. So far, limited attempts have been made to understand the underlying molecular events of the zinc disturbances in PCOS.

Investigating the expression of the zinc transporter ZIP14 in adipose tissue, we found that ZIP14 expression decreased in obese women with and without PCOS. Though, there was no statistically significant effect of PCOS itself on ZIP14 expression. This correlation between the body weight and ZIP14 expression supports our previous study of ZIP14 in adipose tissue from otherwise healthy men and women, in which we found that ZIP14 is downregulated in subcutaneous fat in response to obesity and that the downregulation is reversed by weight loss (27). Downregulation of ZIP14 in a chronic condition with lowgrade inflammation such as obesity might appear paradoxical, as murine studies have shown an upregulation of Zip14 expression in liver, muscle, and adipose tissue after exposure to the pro-inflammatory lipopolysaccharide $(29,49)$. Studies in Zip14 knockout mice have linked lack of this specific transporter to inflammation, with evidence of endotoxemia and increased levels of circulating pro-inflammatory cytokines (29).

Moreover, Zip14 knockouts have increased amounts of body fat and display hypertrophic adipocytes with low differentiation potential in adipose tissues (29). Studies of 3T3-L1 pre-adipocytes confirm that Zip14 is associated with adipocyte differentiation (27-29), and that mobilization of intracellular zinc is decreased in adipocytes with downregulated Zip14 expression (29).

The downregulation of Zip14 in adipocytes is speculated to result in a lack of free cytoplasmic zinc in adipocytes, defined as "the zinc trap" (29) by Troche and coworkers. The zinc trap refers to the fact that downregulation of Zip14 in 3T3-L1 adipocytes increases vesicular zinc yet reduces metallothionein expression (29). As metallothionein expression is tightly regulated by cytoplasmic zinc ions such low expression of metallothionein is considered indicative of a low concentration of free cytoplasmic zinc (50).

Although the present study employs fatty tissue samples as a whole-which includes macrophage involvement-our findings support a condition of cytoplasmic zinc deficiency within adipocytes in obesity, as we see downregulation of ZIP14 paralleled by an upregulation of ZNT1, the only SLC30A efflux transporter in the plasma membrane (51). Supporting an obesity-related zinc dyshomeostasis, a reduced expression of ZIP1-8 and ZNT 2, 3, 6 , and 8 has previously been described in subcutaneous fat from obese individuals (52). However, such changes in zinc homeostasis are likely to be tissue specific. We recently reported decreased
ZNT1 and ZNT6 expression in the human frontal cortex with increased BMI (53), and differences have been noted when comparing subcutaneous and visceral adipose tissues $(52,54)$. An altered expression of zinc transporters indicates a change in the pico-molar concentration and distribution of free intracellular zinc, but it is questionable if it will result in a measurable change in total zinc concentration within the range of a few 100 micromolar. We have previously shown comparable levels of total zinc in adipose tissue from diabetic and non-diabetic sand rats on a high energy diet, as well as in blood samples from obese and non-obese individuals, despite changes in the expression profile of zinc transporters in adipose tissue $(27,54)$. Ultra-structural analysis of fresh tissue samples using the highly sensitive autometallographic technique might in the future provide valuable insights on the in situ location of free or loosely bound zinc (55); however, this method of silver amplification cannot be used for quantitative measurements.

The adipogenic transcription factor PPARG (56) links with PCOS, as specific PPARG gene polymorphisms associate with a reduced risk of PCOS and a better metabolic profile in PCOS women $(31,32)$. PPARG agonists are also used therapeutically in PCOS as it improves menstrual cyclicity, hormone levels, and glucose homeostasis (57). We observed that both obesity and PCOS reduce PPARG expression. Studies in adipocyte cultures, human biopsies, and tissues from Zip14 knockout mice have all shown a positive association between PPARG and ZIP14 expression in adipose tissue $(27,29)$. Thus, Zip 14 knockout mice display a decreased PPARG level and increased adipose tissue hypertrophy (29), indicating that the lack of free cytoplasmic zinc affects PPARG levels in these mice. Accordingly, free cytoplasmic zinc participates in cell differentiation by regulating different signaling pathways and transcription factors (58). Moreover, PPARG signaling and expression is decreased upon zinc deficiency and PPARG structurally requires zinc ions $(33,34)$. Therefore, it is likely that the PPARG downregulation occurring in obese individuals is caused by the decrease in ZIP14 and upregulation of ZNT1. We found no synergistic effect between obesity and PCOS with regard to $P P A R G$ expression. In a larger study, however, it cannot be excluded that an association exists. PPARG expression was significantly reduced in lean women with PCOS, despite an unaltered ZIP14 expression level, compared to lean controls, indicating that another signaling pathway could affect $P P A R G$ in PCOS. The high androgen level in PCOS women could account for some of the PPARG alterations, as androgens can decrease PPARG expression, as well as adipogenesis $(59,60)$.

Similar to ZIP14, GLUT4 and RBP4 expression are reduced in obesity. A statistically significant synergistic effect of PCOS and obesity was found for $R B P 4$, and although not statistically significant for GLUT4, the lean controls appeared to be substantially different from both PCOS groups with regards to GLUT4 expression. The obesity-related changes in these two genes could partly reflect a reduction in free cytoplasmic zinc. GLUT4 levels are downregulated in an obese insulin-resistant state (61) and regulated by the insulin signaling pathway (62), in which free intracellular zinc regulates several signaling steps (63). Zinc can also affect the transport/release of retinol and alter RBP4 levels in liver $(64,65)$. RBP4 has been speculated to be involved in 
inflammation and insulin resistance in obesity and type 2 diabetes (66), but the exact role and regulation in obesity is so far unclear (67). Increased RBP4 levels have been reported in adipose tissue from obese PCOS women vs. obese controls (68). In this study, $R B P 4$ expression was higher in the obese PCOS group vs. obese controls, although the change was not statistically significant with the present sample size.

ZIP14 seem to affect glucose metabolism as Zip14 knockout mice display hyperinsulinemia, augmented insulin receptor phosphorylation, and increased liver glucose (49, 69). ZIP14 might thus affect the symptomatology of PCOS via organs other than white fatty tissue. In this study, ZIP14 expression in subcutaneous fat associates with markers of glucose metabolism in both blood and adipose tissue, although not as significantly as our previous findings in which the HOMA index, fasting glucose, and insulin correlated with ZIP14 expression. However, our previous study included subjects of both sexes which had a higher average BMI in the obese group (27). As for ZIP14, the expression of the counter-regulated influx transporter ZNT1 also showed significant correlations with markers of glucose homeostasis, further linking alterations in intracellular zinc to glucose metabolism.

Sex hormones regulate zinc transporters of the LIV-1 subfamily, and ZIP14 expression can be induced by estrogen (26). However, we found no evidence that the specific hormonal changes occurring in PCOS affect ZIP14 expression in adipose tissue. Furthermore, there were no changes in ZIP9 expression, even though this transporter can function as an androgen receptor (37). Likely, obesity has a stronger influence on zinc homeostasis than the altered hormonal levels in PCOS.

In conclusion, PCOS is a complex disease that involves a zinc homeostatic disturbance and adipocyte dysfunction. The zinc transporters ZNT1 and ZIP14 show an inverse regulation

\section{REFERENCES}

1. March WA, Moore VM, Willson KJ, Phillips DI, Norman RJ, Davies MJ. The prevalence of polycystic ovary syndrome in a community sample assessed under contrasting diagnostic criteria. Hum Reprod (2010) 25:544-51. doi:10.1093/humrep/dep399

2. Fauser BC, Tarlatzis BC, Rebar RW, Legro RS, Balen AH, Lobo R, et al. Consensus on women's health aspects of polycystic ovary syndrome (PCOS): the Amsterdam ESHRE/ASRM-Sponsored 3rd PCOS Consensus Workshop Group. Fertil Steril (2012) 97:28-38.e25. doi:10.1016/j.fertnstert.2011.09.024

3. Kurdoglu Z, Kurdoglu M, Demir H, Sahin HG. Serum trace elements and heavy metals in polycystic ovary syndrome. Hum Exp Toxicol (2012) 31:452-6. doi:10.1177/0960327111424299

4. Chakraborty P, Ghosh S, Goswami SK, Kabir SN, Chakravarty B, Jana K. Altered trace mineral milieu might play an aetiological role in the pathogenesis of polycystic ovary syndrome. Biol Trace Elem Res (2013) 152:9-15. doi:10.1007/s12011-012-9592-5

5. Guler I, Himmetoglu O, Turp A, Erdem A, Erdem M, Onan MA, et al. Zinc and homocysteine levels in polycystic ovarian syndrome patients with insulin resistance. Biol Trace Elem Res (2014) 158:297-304. doi:10.1007/ s12011-014-9941-7

6. Foroozanfard F, Jamilian M, Jafari Z, Khassaf A, Hosseini A, Khorammian $\mathrm{H}$, et al. Effects of zinc supplementation on markers of insulin resistance and lipid profiles in women with polycystic ovary syndrome: a randomized, double-blind, placebo-controlled trial. Exp Clin Endocrinol Diabetes (2015) 123:215-20. doi:10.1055/s-0035-1548790 in subcutaneous adipose tissue in obesity, as well as in obese women with PCOS. The downregulation of ZIP14 expression and the upregulation of ZNT1 expression in obese subjects are indicative of a reduction in free cytoplasmic zinc, with potential down-stream effects on PPARG, RBP4, and GLUT4 expression, as well as RBP4 appear to be directly involved in PCOS pathology.

\section{AUTHOR CONTRIBUTIONS}

TM, PS, AL, KS, and JL contributed to the measurements and analyses. KS, AL, JR, SP, and BB supervised TM when writing the manuscript and when designing the study. All the authors took part in the construction of the manuscript and have read and approved the final manuscript.

\section{ACKNOWLEDGMENTS}

The authors kindly thank Pia Hornbek, Lenette Pedersen, and Søren Paulsen for technical assistance.

\section{FUNDING}

The authors thank the Faculty of Health Science, Aarhus University, The A.P. Møller Foundation for the Advancement of Medical Science, and The Margrethe Møller Foundation for financially supporting this study.

\section{SUPPLEMENTARY MATERIAL}

The Supplementary Material for this article can be found online at http://journal.frontiersin.org/article/10.3389/fendo.2017.00038/ full\#supplementary-material.
7. Al-Maroof RA, Al-Sharbatti SS. Serum zinc levels in diabetic patients and effect of zinc supplementation on glycemic control of type 2 diabetics. Saudi Med J (2006) 27:344-50.

8. de Luis DA, Pacheco D, Izaola O, Terroba MC, Cuellar L, Cabezas G. Micronutrient status in morbidly obese women before bariatric surgery. Surg Obes Relat Dis (2013) 9:323-7. doi:10.1016/j.soard.2011.09.015

9. de Luis DA, Pacheco D, Izaola O, Terroba MC, Cuellar L, Martin T. Zinc and copper serum levels of morbidly obese patients before and after biliopancreatic diversion: 4 years of follow-up. J Gastrointest Surg (2011) 15:2178-81. doi:10.1007/s11605-011-1647-y

10. Aguilar MV, Saavedra P, Arrieta FJ, Mateos CJ, Gonzalez MJ, Meseguer I, et al. Plasma mineral content in type- 2 diabetic patients and their association with the metabolic syndrome. Ann Nutr Metab (2007) 51:402-6. doi:10.1159/000108108

11. Costarelli L, Muti E, Malavolta M, Cipriano C, Giacconi R, Tesei S, et al. Distinctive modulation of inflammatory and metabolic parameters in relation to zinc nutritional status in adult overweight/obese subjects. J Nutr Biochem (2010) 21:432-7. doi:10.1016/j.jnutbio.2009.02.001

12. Vallee BL, Falchuk KH. The biochemical basis of zinc physiology. Physiol Rev (1993) 73:79-118.

13. Hogstrand C, Zheng D, Feeney G, Cunningham P, Kille P. Zinc-controlled gene expression by metal-regulatory transcription factor 1 (MTF1) in a model vertebrate, the zebrafish. Biochem Soc Trans (2008) 36:1252-7. doi:10.1042/BST0361252

14. Haase H, Maret W. Fluctuations of cellular, available zinc modulate insulin signaling via inhibition of protein tyrosine phosphatases. J Trace Elem Med Biol (2005) 19:37-42. doi:10.1016/j.jtemb.2005.02.004 
15. Vardatsikos G, Pandey NR, Srivastava AK. Insulino-mimetic and antidiabetic effects of zinc. J Inorg Biochem (2013) 120:8-17. doi:10.1016/j. jinorgbio.2012.11.006

16. Coulston L, Dandona P. Insulin-like effect of zinc on adipocytes. Diabetes (1980) 29:665-7. doi:10.2337/diab.29.8.665

17. Ezaki O. IIb group metal ions $\left(\mathrm{Zn}^{2+}, \mathrm{Cd}^{2+}, \mathrm{Hg}^{2+}\right)$ stimulate glucose transport activity by post-insulin receptor kinase mechanism in rat adipocytes. J Biol Chem (1989) 264:16118-22.

18. Halsted JA, Ronaghy HA, Abadi P, Haghshenass M, Amirhakemi GH, Barakat RM, et al. Zinc deficiency in man. The Shiraz experiment. Am J Med (1972) 53:277-84. doi:10.1016/0002-9343(72)90169-6

19. Salgueiro MJ, Weill R, Zubillaga M, Lysionek A, Caro R, Goldman C, et al. Zinc deficiency and growth: current concepts in relationship to two important points: intellectual and sexual development. Biol Trace Elem Res (2004) 99:49-69. doi:10.1385/BTER:99:1-3:049

20. Prasad AS, Halsted JA, Nadimi M. Syndrome of iron deficiency anemia, hepatosplenomegaly, hypogonadism, dwarfism and geophagia. Am J Med (1961) 31:532-46. doi:10.1016/0002-9343(61)90137-1

21. King JC. Determinants of maternal zinc status during pregnancy. Am J Clin Nutr (2000) 71:1334S-43S.

22. Golub MS, Keen CL, Gershwin ME, Styne DM, Takeuchi PT, Ontell F, et al. Adolescent growth and maturation in zinc-deprived rhesus monkeys. Am J Clin Nutr (1996) 64:274-82.

23. Favier AE. The role of zinc in reproduction. Hormonal mechanisms. Biol Trace Elem Res (1992) 32:363-82. doi:10.1007/BF02784623

24. Huang L, Tepaamorndech S. The SLC30 family of zinc transporters - a review of current understanding of their biological and pathophysiological roles. Mol Aspects Med (2013) 34:548-60. doi:10.1016/j.mam.2012.05.008

25. Jeong J, Eide DJ. The SLC39 family of zinc transporters. Mol Aspects Med (2013) 34:612-9. doi:10.1016/j.mam.2012.05.011

26. Taylor KM, Morgan HE, Smart K, Zahari NM, Pumford S, Ellis IO, et al. The emerging role of the LIV-1 subfamily of zinc transporters in breast cancer. Mol Med (2007) 13:396-406. doi:10.2119/2007-00040.Taylor

27. Maxel T, Smidt K, Larsen A, Bennetzen M, Cullberg K, Fjeldborg K, et al. Gene expression of the zinc transporter ZIP14 (SLC39a14) is affected by weight loss and metabolic status and associates with PPARgamma in human adipose tissue and 3T3-L1 pre-adipocytes. BMC Obes (2015) 2:46. doi:10.1186/ s40608-015-0076-y

28. Tominaga K, Kagata T, Johmura Y, Hishida T, Nishizuka M, Imagawa M. SLC39A14, a LZT protein, is induced in adipogenesis and transports zinc. FEBS J (2005) 272:1590-9. doi:10.1111/j.1742-4658.2005.04580.x

29. Troche C, Aydemir TB, Cousins RJ. Zinc transporter Slc39a14 regulates inflammatory signaling associated with hypertrophic adiposity. Am J Physiol Endocrinol Metab (2015) 310:E258-68. doi:10.1152/ajpendo.00421.2015

30. Janani C, Ranjitha Kumari BD. PPAR gamma gene - a review. Diabetes Metab Syndr (2015) 9:46-50. doi:10.1016/j.dsx.2014.09.015

31. Zhang H, Bi Y, Hu C, Lu W, Zhu D. Association between the Pro12Ala polymorphism of PPAR-gamma gene and the polycystic ovary syndrome: a meta-analysis of case-control studies. Gene (2012) 503:12-7. doi:10.1016/j. gene.2012.04.083

32. Knebel B, Lehr S, Janssen OE, Hahn S, Nitzgen U, Jacob S, et al. Genetic variants in central metabolic genes influence some but not all relations of inflammatory markers in a collective with polycystic ovary syndrome. Arch Physiol Biochem (2012) 118:219-29. doi:10.3109/13813455.2012.697903

33. Meerarani P, Reiterer G, Toborek M, Hennig B. Zinc modulates PPARgamma signaling and activation of porcine endothelial cells. J Nutr (2003) 133:3058-64.

34. Owen GI, Zelent A. Origins and evolutionary diversification of the nuclear receptor superfamily. Cell Mol Life Sci (2000) 57:809-27. doi:10.1007/ s000180050043

35. el-Tanani MK, Green CD. Insulin/IGF-1 modulation of the expression of two estrogen-induced genes in MCF-7 cells. Mol Cell Endocrinol (1996) 121:29-35. doi:10.1016/0303-7207(96)03844-0

36. Taylor KM, Nicholson RI. The LZT proteins; the LIV-1 subfamily of zinc transporters. Biochim Biophys Acta (2003) 1611:16-30. doi:10.1016/ S0005-2736(03)00048-8

37. Pascal LE, Wang Z. Unzipping androgen action through ZIP9: a novel membrane androgen receptor. Endocrinology (2014) 155:4120-3. doi:10.1210/ en.2014-1749
38. Thomas P, Pang Y, Dong J, Berg AH. Identification and characterization of membrane androgen receptors in the ZIP9 zinc transporter subfamily: II. Role of human ZIP9 in testosterone-induced prostate and breast cancer cell apoptosis. Endocrinology (2014) 155:4250-65. doi:10.1210/ en.2014-1201

39. Azziz R. Diagnosis of polycystic ovarian syndrome: the Rotterdam criteria are premature. JClin Endocrinol Metab (2006) 91:781-5. doi:10.1210/ jc.2005-2153

40. Svendsen PF, Nilas L, Norgaard K, Jensen JE, Madsbad S. Obesity, body composition and metabolic disturbances in polycystic ovary syndrome. Hum Reprod (2008) 23:2113-21. doi:10.1093/humrep/den211

41. Svendsen PF, Madsbad S, Nilas L, Paulsen SK, Pedersen SB. Expression of 11beta-hydroxysteroid dehydrogenase 1 and 2 in subcutaneous adipose tissue of lean and obese women with and without polycystic ovary syndrome. Int J Obes (Lond) (2009) 33:1249-56. doi:10.1038/ijo.2009.165

42. Svendsen PF, Nilas L, Madsbad S, Holst JJ. Incretin hormone secretion in women with polycystic ovary syndrome: roles of obesity, insulin sensitivity, and treatment with metformin. Metabolism (2009) 58:586-93. doi:10.1016/j. metabol.2008.11.009

43. Svendsen PF, Madsbad S, Nilas L. The insulin-resistant phenotype of polycystic ovary syndrome. Fertil Steril (2010) 94:1052-8. doi:10.1016/j. fertnstert.2009.04.008

44. Svendsen PF, Christiansen M, Hedley PL, Nilas L, Pedersen SB, Madsbad S. Adipose expression of adipocytokines in women with polycystic ovary syndrome. Fertil Steril (2012) 98:235-41. doi:10.1016/j.fertnstert.2012.03.056

45. Matthews DR, Hosker JP, Rudenski AS, Naylor BA, Treacher DF, Turner RC. Homeostasis model assessment: insulin resistance and beta-cell function from fasting plasma glucose and insulin concentrations in man. Diabetologia (1985) 28:412-9. doi:10.1007/BF00280883

46. Volund A. Conversion of insulin units to SI units. Am J Clin Nutr (1993) 58:714-5.

47. Gabrielsson BG, Olofsson LE, Sjogren A, Jernas M, Elander A, Lonn M, et al. Evaluation of reference genes for studies of gene expression in human adipose tissue. Obes Res (2005) 13:649-52. doi:10.1038/oby.2005.72

48. Jamilian M, Foroozanfard F, Bahmani F, Talaee R, Monavari M, Asemi Z. Effects of zinc supplementation on endocrine outcomes in women with polycystic ovary syndrome: a randomized, double-blind, placebo-controlled trial. Biol Trace Elem Res (2016) 170:271-8. doi:10.1007/s12011-015-0480-7

49. Beker AT, Chang SM, Guthrie GJ, Maki AB, Ryu MS, Karabiyik A, et al. Zinc transporter ZIP14 functions in hepatic zinc, iron and glucose homeostasis during the innate immune response (endotoxemia). PLoS One (2012) 7:e48679. doi:10.1371/journal.pone.0048679

50. Maret W. Metals on the move: zinc ions in cellular regulation and in the coordination dynamics of zinc proteins. Biometals (2011) 24:411-8. doi:10.1007/ s10534-010-9406-1

51. Kambe T, Yamaguchi-Iwai Y, Sasaki R, Nagao M. Overview of mammalian zinc transporters. Cell Mol Life Sci (2004) 61:49-68. doi:10.1007/ s00018-003-3148-y

52. Smidt K, Pedersen SB, Brock B, Schmitz O, Fisker S, Bendix J, et al. Zinctransporter genes in human visceral and subcutaneous adipocytes: lean versus obese. Mol Cell Endocrinol (2007) 264:68-73. doi:10.1016/j.mce.2006.10.010

53. Olesen RH, Hyde TM, Kleinman JE, Smidt K, Rungby J, Larsen A. Obesity and age-related alterations in the gene expression of zinc-transporter proteins in the human brain. Transl Psychiatry (2016) 6:e838. doi:10.1038/tp.2016.83

54. Maxel T, Pold R, Larsen A, Pedersen SB, Carlson D, Rolin B, et al. Dysregulation of zinc and iron balance in adipose tissue from diabetic sand rats (Psammomys obesus). J Diabetes Metab (2015) 6:497.

55. Danscher G, Stoltenberg M. Silver enhancement of quantum dots resulting from (1) metabolism of toxic metals in animals and humans, (2) in vivo, in vitro and immersion created zinc-sulphur/zinc-selenium nanocrystals, (3) metal ions liberated from metal implants and particles. Prog Histochem Cytochem (2006) 41:57-139. doi:10.1016/j.proghi.2006.06.001

56. Rosen ED, MacDougald OA. Adipocyte differentiation from the inside out. Nat Rev Mol Cell Biol (2006) 7:885-96. doi:10.1038/nrm2066

57. Rautio K, Tapanainen JS, Ruokonen A, Morin-Papunen LC. Endocrine and metabolic effects of rosiglitazone in overweight women with PCOS: a randomized placebo-controlled study. Hum Reprod (2006) 21:1400-7. doi:10.1093/ humrep/dei505 
58. Beyersmann D, Haase H. Functions of zinc in signaling, proliferation and differentiation of mammalian cells. Biometals (2001) 14:331-41. doi:10.102 3/A:1012905406548

59. Chazenbalk G, Singh P, Irge D, Shah A, Abbott DH, Dumesic DA. Androgens inhibit adipogenesis during human adipose stem cell commitment to preadipocyte formation. Steroids (2013) 78:920-6. doi:10.1016/j.steroids.2013.05.001

60. Wang YX, Zhu WJ, Xie BG. Expression of PPAR-gamma in adipose tissue of rats with polycystic ovary syndrome induced by DHEA. Mol Med Rep (2014) 9:889-93. doi:10.3892/mmr.2014.1895

61. Abel ED, Peroni O, Kim JK, Kim YB, Boss O, Hadro E, et al. Adipose-selective targeting of the GLUT4 gene impairs insulin action in muscle and liver. Nature (2001) 409:729-33. doi:10.1038/35055575

62. Huang S, Czech MP. The GLUT4 glucose transporter. Cell Metab (2007) 5:237-52. doi:10.1016/j.cmet.2007.03.006

63. Rutter GA, Chabosseau P, Bellomo EA, Maret W, Mitchell RK, Hodson DJ, et al. Intracellular zinc in insulin secretion and action: a determinant of diabetes risk? Proc Nutr Soc (2016) 75:61-72. doi:10.1017/S0029665115003237

64. Mobarhan S, Greenberg B, Mehta R, Friedman H, Barch D. Zinc deficiency reduces hepatic cellular retinol-binding protein in rats. Int J Vitam Nutr Res (1992) 62:148-54.

65. Boron B, Hupert J, Barch DH, Fox CC, Friedman H, Layden TJ, et al. Effect of zinc deficiency on hepatic enzymes regulating vitamin A status. J Nutr (1988) 118:995-1001.

66. Zabetian-Targhi F, Mahmoudi MJ, Rezaei N, Mahmoudi M. Retinol binding protein 4 in relation to diet, inflammation, immunity, and cardiovascular diseases. Adv Nutr (2015) 6:748-62. doi:10.3945/an.115.008292
67. Takebayashi K, Aso Y, Inukai T. Role of retinol-binding protein 4 in the pathogenesis of type 2 diabetes. Expert Rev Endocrinol Metab (2008) 3:161-73. doi:10.1586/17446651.3.2.161

68. Tan BK, Chen J, Lehnert H, Kennedy R, Randeva HS. Raised serum, adipocyte, and adipose tissue retinol-binding protein 4 in overweight women with polycystic ovary syndrome: effects of gonadal and adrenal steroids. JClin Endocrinol Metab (2007) 92:2764-72. doi:10.1210/ jc. 2007-0091

69. Hojyo S, Fukada T, Shimoda S, Ohashi W, Bin BH, Koseki H, et al. The zinc transporter SLC39A14/ZIP14 controls G-protein coupled receptor-mediated signaling required for systemic growth. PLoS One (2011) 6:e18059. doi:10.1371/journal.pone.0018059

Conflict of Interest Statement: The authors declare that they have no conflict of interest as the research was conducted in the absence of any commercial or financial relationships that could be construed as a potential conflict of interest.

Copyright (C) 2017 Maxel, Svendsen, Smidt, Lauridsen, Brock, Pedersen, Rungby and Larsen. This is an open-access article distributed under the terms of the Creative Commons Attribution License (CC BY). The use, distribution or reproduction in other forums is permitted, provided the original author(s) or licensor are credited and that the original publication in this journal is cited, in accordance with accepted academic practice. No use, distribution or reproduction is permitted which does not comply with these terms. 